\title{
Hepatocellular carcinoma occurred in a Hepatitis B carrier clinic cohort during a mean follow up of 10 years
}

\author{
Cheng-Jen Chen ${ }^{1}$, Jennifer Tai ${ }^{2}$, Dar-In Tai ${ }^{1,2}$ \\ 'Liver Research Center, Chang Gung Memorial Hospital, Linkou Medical Center, Taoyuan 33305, Taiwan. \\ ${ }^{2}$ Department of Internal Medicine, Chang Gung Memorial Hospital, Linkou Medical Center, Taoyuan 33305, Taiwan.
}

Correspondence to: Dr. Dar-In Tai, Liver Research Center, Chang Gung Memorial Hospital and Chang Gung University College of Medicine, 199 Tung-Hwa North Road, Taipei 10591, Taiwan. E-mail: tai48978@cgmh.org.tw; imjenkid@gmail.com; k85731@cgmh.org.tw

\begin{abstract}
How to cite this article: Chen CJ, Tai J, Tai DI. Hepatocellular carcinoma occurred in a Hepatitis B carrier clinic cohort during a mean follow up of 10 years. Hepatoma Res 2019;5:25. http://dx.doi.org/10.20517/2394-5079.2019.12
\end{abstract}

Received: 17 Feb 2019 First Decision: 7 Mar 2019 Revised: 12 Apr 2019 Accepted: 18 Apr 2019 Published: 11 Jul 2019

Science Editor: Jin-Lin Hou Copy Editor: Cai-Hong Wang Production Editor: Jing Yu

\begin{abstract}
Aim: Chronic persistent hepatitis B virus carriers are generally asymptomatic until the advanced stage of the disease. The "Hepatitis B-Carrier Clinics" of Chang Gung Memorial Hospital has been using alpha-fetoprotein (AFP) and liver ultrasound for early detection of hepatocellular carcinoma $(\mathrm{HCC})$ in hepatitis B surface antigen ( $\mathrm{HBs} \mathrm{Ag}$ ) carriers since 1980.
\end{abstract}

Methods: We evaluated the results of surveillance between 1980 and 2012 by collecting clinic data, matched cancer registry status, and national mortality database status.

Results: Of 15,235 HBsAg carriers, 238 instances of HCC (1.5\% or 156.2/100,000 person-years) were detected over a mean follow-up period of $10.0 \pm 7.6$ years. There were more men $(89.1 \%)$ and patients with liver cirrhosis $(70.2 \%)$ in the HCC group $(P<0.001)$, and both the initial and maximal alanine aminotransferase (ALT) levels were higher in this group $(P<0.001)$. One hundred and thirty cases of HCC $(54.6 \%)$ were identified during regular follow-up sessions, 55 (23.1\%) were detected after the regular schedule had lapsed ("out-of-schedule"), and 53 (22.3\%) were lost to follow-up completely. The mean tumor size was smaller in the regular group than in the outof-schedule group $(2.72 \mathrm{~cm} v s .4 .59 \mathrm{~cm}, P<0.001)$, and the survival rate was higher $(43.8 \% v s .30 .9 \%, P<0.001)$.

Conclusion: The incidence of HCC was relatively low in the HBsAg-Carrier Clinics cohort. Surveillance for early

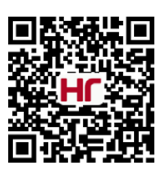


diagnosis of HCC improved the survival of high-risk HBsAg carriers. To ensure cost-effectiveness, we suggest using different screening strategies according to the individual risk of hepatocarcinogenesis.

Keywords: Hepatitis B surface antigen, retrospective cohort, hepatocellular carcinoma, surveillance

\section{INTRODUCTION}

Chronic persistent hepatitis B virus (HBV) infection is a global disease, and its prevalence is highest in Africa and East Asia ${ }^{[1-4]}$. The chronic relapsing inflammation caused by HBV leads to chronic hepatitis, liver cirrhosis, and hepatocellular carcinoma (HCC $)^{[4]}$. Patients with HBV are generally asymptomatic and unaware of their illness until they reach the advanced stage. Early diagnosis of HCC through periodic surveillance has become one of the strategies for management of chronic hepatitis B surface antigen (HBsAg) carriers ${ }^{[5-7]}$. Diagnosis of HCC in the early, treatable stages leads to improved survival ${ }^{[7,8]}$. This is especially important for areas with endemic HBV infection ${ }^{[1-3,9]}$. The methods for early detection of HCC mainly rely on alpha-fetoprotein (AFP), AFP-lectin 3 fraction (AFP-L3), des- $\gamma$-carboxy prothrombin, and liver ultrasound (US) ${ }^{[5-8,10,11]}$. Many new biomarkers are under investigation ${ }^{[12-14]}$, but they appear to be more expensive and were not found to be significantly superior to AFP and US in a large population survey ${ }^{[15]}$.

In addition to detection tools, a number of other variables, including duration of surveillance, individual risk, and patient attitude, may also contribute to the effectiveness of surveillance. We have been performing surveillance for early detection of HCC in a hepatitis B carrier clinic for more than 30 years ${ }^{[5,6]}$. This analysis was conducted to assess our results and to improve our surveillance strategy.

\section{METHODS}

The HBsAg-Carrier Clinics of Chang Gung Memorial Hospital in Taipei and Linkou Medical Centers have been in operation since 1980, and they provide an easily accessible service for chronic HBsAg carriers in Taiwan. Most patients who visit the HBsAg-Carrier Clinics are asymptomatic upon entry. They visit the clinics because the presence of HBsAg has been incidentally detected upon blood donation, in a general check-up, or in a work-up for a non-liver-related disease, or they are referred from our outpatient department as stable HBsAg carriers with normal alanine aminotransferase (ALT).

A total of 15,235 HBsAg carriers with persistent HBsAg for more than six months had been registered in the HBsAg-Carrier Clinics by 2012. Patients with dual infections were excluded. The subjects underwent 275,324 visits and had a mean follow-up duration of $10.0 \pm 7.65$ years. Upon registration with the clinic, the subjects underwent liver biochemical tests and testing for serologic markers of hepatitis viruses, AFP, and real-time liver US. After this initial visit, the subjects were followed-up every 3-12 months, with ALT, AFP, and US as the basic measures.

This HBsAg-Carrier Clinics has three full-time clinic staffs and a private line telephone to arrange registration and visits. The clinic staffs recorded basic information, delivered patient education, collected data from each visit, and entered these data into the hospital's main computer. One of the main aims of this clinic is to detect HCC at the early stage. For patients unable to keep up with the follow-up schedule, reminder letters are sent.

In this study, we examined data from the HBsAg-Carrier Clinic from 1980-2012. This long-term follow-up analysis of chronic HBsAg carriers was approved by the human research committee of Chang Gung Memorial Hospital (IRB No: 201600523B0). 


\section{Identification of hepatocellular carcinoma}

One of the main aims of this study was to determine the incidence of HCC in this cohort. Diagnosis of HCC was ascertained based on cytology or histology reports. We also included subjects with at least two findings from the AFP, liver US, computer tomography (CT), magnetic resonance imaging (MRI), and angiography tests indicating they were positive for $\mathrm{HCC}^{[16]}$. In addition, disease progression based on one imagingmodality study was accepted as a diagnosis of HCC.

In addition to the medical records maintained in this clinic, the study subjects had linked records in the Cancer Registration Database of Chang Gung Memorial Hospital. This database contains all information recorded about all cancers diagnosed or treated at this hospital since 1987. We linked our cohort with this database to retrieve all HCC cases identified between 1987 and 2007 in other departments.

The subjects' national identification numbers were also linked with our national mortality database, which has been maintained by the Statistics Office of Taiwan's Department of Health since 1985. Cause of death was classified using the ninth revision of the International Classification of Diseases, Injuries and Causes of Death (ICD-9, World Health Organization, 1977). We matched our cohort with this database between 1985 and 2007. For patients lost to follow-up in our hospital, the national mortality database was the only available source of information regarding diagnosis of HCC.

\section{Classification of HCC according to follow-up schedule}

The patients in this study differed with respect to the point at which HCC was detected during their followup schedule, and they were therefore classified into three groups. The first group comprised patients with HCC identified within one year of last follow-up (regular follow-up group). The second group comprised patients who were lost to follow-up for more than one year but returned with HCC, and those for whom HCC was identified upon initial presentation (out-of-schedule group). The third group comprised patients who were completely lost to follow-up, but who were identified as having died from HCC based on the national mortality database (lost group).

\section{Statistical analysis}

To compare the characteristics of the three groups, the $\chi^{2}$ test, Fisher's exact test, Student's $t$-test, linearby-linear association, Pearson's correlation, and ANOVA were used as appropriate. Survival analysis was done using the Kaplan-Meier actuarial curve method with the log-rank test. Univariate and multivariate analyses were performed to identify factors associated with mortality and HCC or development of cirrhosis. Variables found to be significant in the univariate models were tested in a multivariate setting using the Cox proportional hazards regression model. All statistical analyses were performed using SPSS software (version 22; SPSS Inc., Chicago, IL) and $P<0.05$ was considered significant.

\section{RESULTS}

During the study period, 238 HCC cases were identified (1.5\% or 156.2/100,000 person-years, Table 1). Male sex (89.1\% in the HCC group vs. 58.6\% in the non-HCC HBsAg carriers, $P<0.001)$, age at enrollment $(43.32$ vs. 34.96 years, $P<0.001)$, prevalence of liver cirrhosis $(70.2 \% v s .2 .7 \%, P<0.001)$, and initial ALT (123.72 vs. 63.22 U/L, $P<0.001)$ and maximal ALT levels (310.73vs. $130.63 \mathrm{U} / \mathrm{L}, P<0.001)$ were significantly associated with HCC [Table 1 and Figure 1]. In the HBsAg carriers without HCC, 72.6\% had initial ALT levels lower than $2 \times$ the upper limit of normal (ULN; Figure 2A) and $69.3 \%$ had a maximal ALT level lower than $5 \times$ ULN [Figure 2B]. In contrast, 63.5\% HCC patients had an initial ALT level greater than or equal two $2 \times$ ULN and $71.5 \%$ had a maximal ALT level greater than or equal to $5 \times$ ULN.

Of the patients with HCC, $130(54.6 \%)$ cases were identified during regular follow-up, 55 (23.1\%) were in the out-of-schedule group, and $53(22.3 \%)$ cases were lost to follow-up [Table 2]. The diagnosis was established 
Table 1. Demographics of chronic HBsAg carriers with and without HCC

\begin{tabular}{lllll}
\hline & \multicolumn{2}{c}{ HBsAg carriers } & \multicolumn{2}{c}{ P value } \\
\cline { 2 - 5 } & \multicolumn{1}{c}{ Without HCC } & HCC & Univariate & Multivariate \\
\hline Total & 14,997 & 238 & & \\
Male & $8,793(58.6 \%)$ & $212(89.1 \%)$ & $<0.001$ & $<0.001$ \\
Age at enrollment (years) & $34.96 \pm 10.08$ & $43.32 \pm 11.18$ & $<0.001$ & $<0.001$ \\
Liver cirrhosis & $407(2.7 \%)$ & $167(70.2 \%)$ & $<0.001$ & $<0.001$ \\
Initial ALT (U/L) & $63.22 \pm 206.16$ & $123.72 \pm 264.12$ & $<0.001$ & 0.434 \\
Maximal ALT (U/L) & $130.63 \pm 310.75$ & $310.73 \pm 487.70$ & $<0.001$ & $<0.001$ \\
Anti-HBV therapy & $876(5.8 \%)$ & $55(23.1)$ & $<0.001$ & $<0.001$ \\
Thymosin & $13(0.08 \%)$ & $1(0.4 \%)$ & & \\
IFN/pegIFN & $130(0.8 \%)$ & $6(2.5 \%)$ & & \\
LAM/ADV/TBV & $342(2.3 \%)$ & $31(13.0 \%)$ & & \\
ETV/TDF & $386(2.6 \%)$ & $17(7.1 \%)$ & & \\
Follow-up (years) & $9.96 \pm 7.66$ & $12.45 \pm 6.23$ & $<0.001$ & \\
\hline
\end{tabular}

ALT: alanine aminotransferase; ADV: adefovir; ETV: entecavir; IFN: interferon; pegIFN: pegylated interferon; LAM: lamivudine; TBV: telbivudine; TDF: tenofovir

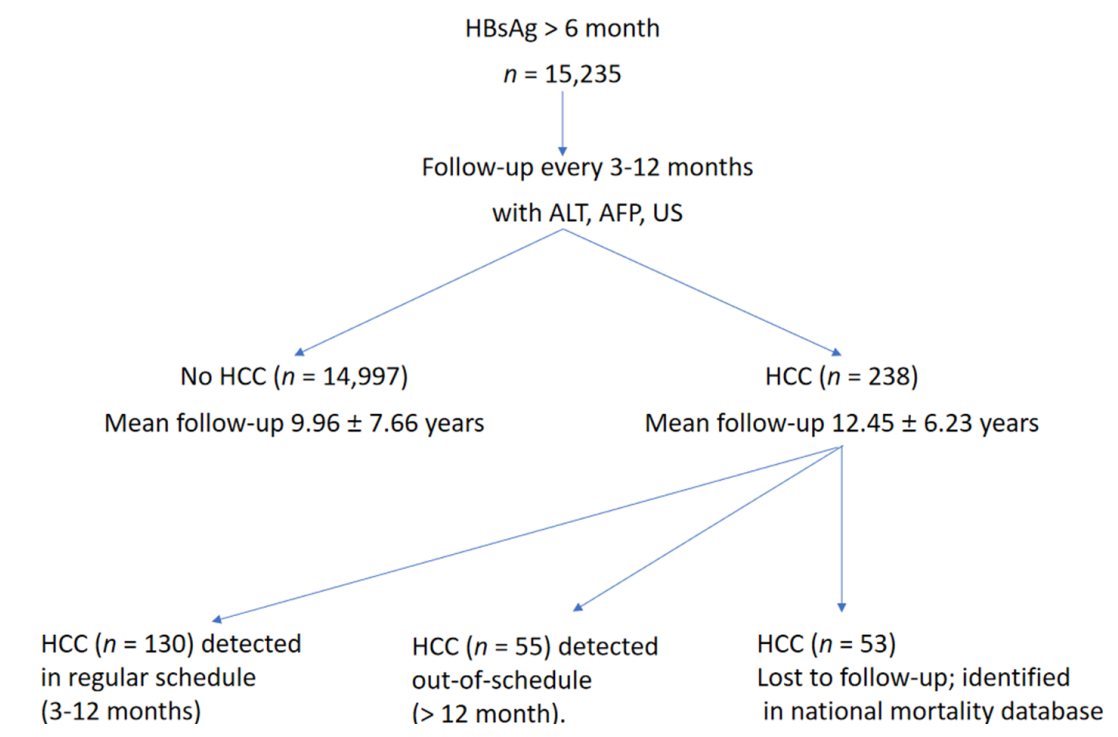

Figure 1. Patient flowchart. AFP: alpha-fetoprotein; ALT: alanine aminotransferase; HCC: hepatocellular carcinoma; US: ultrasound

via histology or cytology in 119 (50\%) patients. The mean tumor size was smaller $(2.72 v s .4 .59 \mathrm{~cm}, P<0.001)$ in the regular follow-up group than in the out-of-schedule group. The regular follow-up group had the highest survival rate $(43.8 \%)$ of the three groups $(P<0.001)$ at end of the study period. There was no difference in age at diagnosis or age at death between the groups.

Of the 130 HCC cases detected during regular follow-up, $47(36.2 \%)$ patients had at least one liver nodule identified via US during the three months before the diagnosis. Sixteen (12.3\%) of these patients had three or more nodules.

During the study period, 931 patients received anti-HBV therapy. The medications administered were: thymosin (14 patients); interferon or pegylated interferon (136 patients); lamivudine, adefovir, or telbivudine (373 patients); and entecavir or tenofovir (403 patients). More patients in the HCC group received anti-HBV therapy than in the non-HCC HBsAg-carrier group (Table 1; $P<0.001$ ). 

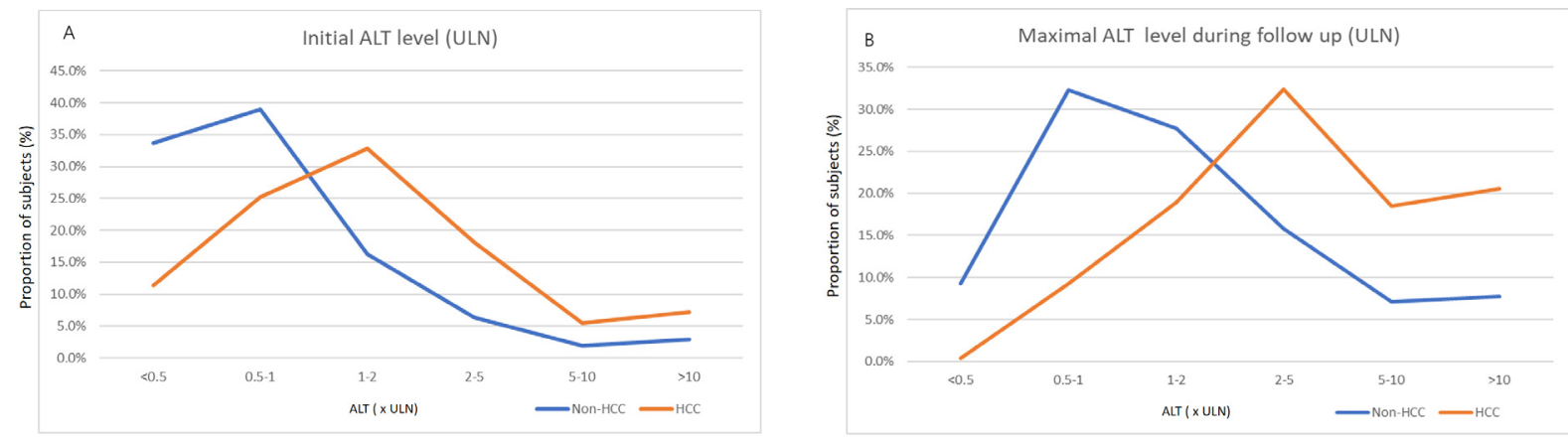

Figure 2. $A$ : the hepatocellular carcinoma (HCC) group had higher initial alanine aminotransferase (ALT) levels than HBsAg carriers without HCC $(P<0.001)$. The majority of HCC cases were patients who had presented with an initial ALT level around $1-2 \times$ the upper limit of normal (ULN), while most of HBsAg carriers without HCC had presented with normal ALT levels; B: the maximal ALT level was higher in HCC cases than in HBsAg carriers without HCC $(P<0.001)$. Most of the HBsAg carriers without HCC had maximal ALT levels below $2 \times$ ULN

Table 2. HCC classification according to follow-up schedule

\begin{tabular}{|c|c|c|c|c|}
\hline & \multicolumn{3}{|c|}{ Follow-up schedule } & \multirow{2}{*}{$P$ value } \\
\hline & Regular & Out-of-schedule & Lost & \\
\hline Total & 130 & 55 & 53 & \\
\hline Male & $115(88.5 \%)$ & $51(92.7 \%)$ & $44(83.0 \%)$ & NS \\
\hline Cirrhosis & $93(71.5 \%)$ & $37(67.3 \%)$ & $?$ & NS \\
\hline \multicolumn{5}{|l|}{ Diagnosis } \\
\hline Histology & $56(43.1 \%)$ & $28(50.9 \%)$ & $?$ & NS \\
\hline Cytology & $27(20.8 \%)$ & $8(14.5 \%)$ & $?$ & \\
\hline Age at diagnosis (years) & $55.8 \pm 10.3$ & $55.2 \pm 11.9$ & $?$ & NS \\
\hline Tumor size $(\mathrm{cm})$ & $2.72 \pm 1.58$ & $4.59 \pm 3.64$ & $?$ & $<0.001$ \\
\hline Survivors & $57(43.8 \%)$ & $17(30.9 \%)$ & $0(0 \%)$ & $<0.001$ \\
\hline Age at death (years) & $61.8 \pm 10.6^{\star}$ & $59.7 \pm 12.6$ & $57.1 \pm 10.3^{\star}$ & $0.035^{\star}$ \\
\hline
\end{tabular}

*Groups for comparison

\section{DISCUSSION}

Only 238 ( $1.5 \%$ or 156.2 person-years) HCC cases were identified among 15,235 HBsAg carriers during a mean follow-up of 10 years. The annual incidence of HCC was $0.53 \%$ in men and $0.04 \%$ in women [Figure 3]. The incidence of HCC in this cohort was relatively low compared with the 826 person-years reported in our previous study of 432 patients with chronic hepatitis $\mathrm{B}^{[5]}$. In general, the incidence in our study is lower than in studies of patients with chronic hepatitis and higher than in studies of inactive carriers ${ }^{[17]}$. In a study examining HCCrisk stratification ${ }^{[18]}$, the 10 -year cumulative risk score for developing HCC was $1.2 \%-2 \%$ when only male sex and subjects aged 50-59 years were considered ${ }^{[18]}$. The HCC diagnostic age in this cohort was around 55 years [Table 2]. Therefore, our HCC incidence (1.5\%/10 years) is within the range of that prediction. HCC screening is cost-effective and is recommended for groups in which annual HCC incidence exceeds $1.5 \%{ }^{[19]}$. Therefore, lifelong screening of all HBsAg carriers for liver cancer would not be cost-effective in this cohort. We do have other reasons for maintaining this clinic, such as screening for chronic hepatitis and liver cirrhosis that require therapy, understanding the history of chronic HBV infection, and others. However, we should focus surveillance efforts on high-risk HBsAg carriers ${ }^{[20]}$. Without complicated parameters, this study has identified male gender (89.1\%), maximal ALT level greater than or equal to $5 \times \operatorname{ULN}(71.5 \%)$, liver cirrhosis (70.2\%), and age over 40 at enrollment (95\%) as risk factors for HCC (Table 1; logistic regression, $P<0.001$ ). Patients who have these clinical and demographic characteristics warrant active surveillance.

A large proportion of the patients were unable to adhere to the follow-up schedule in this cohort. Only $54.6 \%(130 / 238)$ of the HCC cases were identified during the regular follow-up schedule, while $55(23.1 \%)$ 


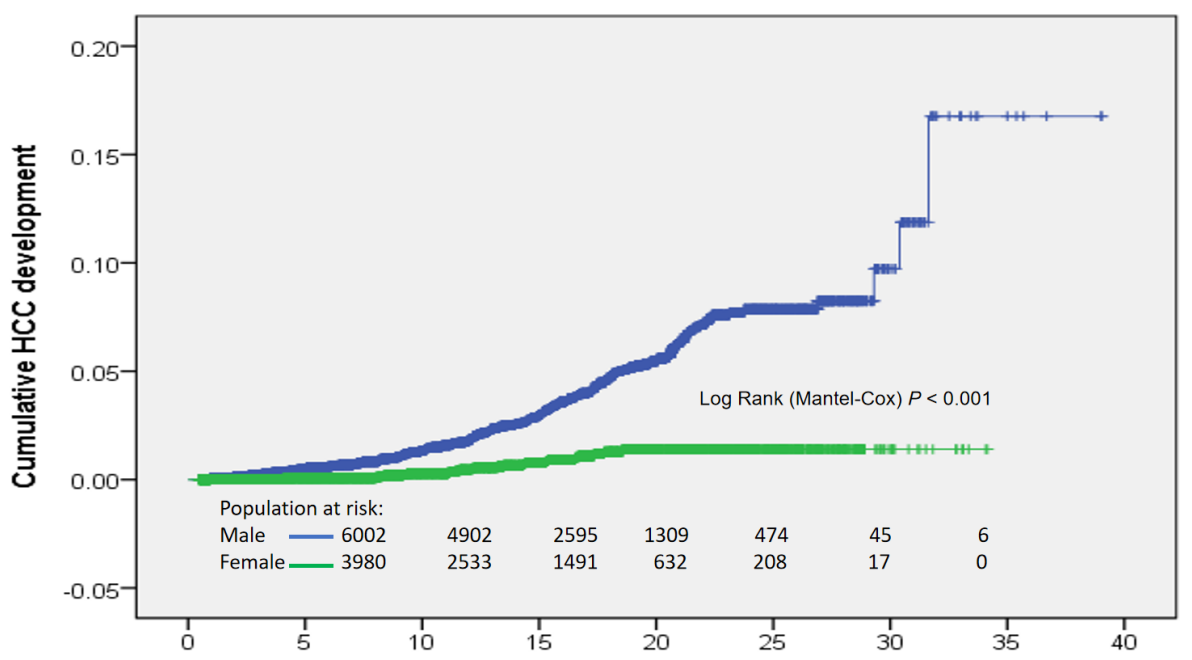

Figure 3. Cumulative hepatocellular carcinoma (HCC) development in men and women. The annual HCC incidence was $0.53 \%$ in men and $0.04 \%$ in women

were patients who returned with HCC and 53 (22.3\%) were patients who had been completely lost to followup. This suggests that ongoing surveillance at 3-12-month intervals was difficult to maintain for nearly half of the patients. The results from our single hospital with 15,235 cases are similar to a meta-analysis of 22 reports covering 19,511 cases $^{[21]}$. In that study, Wang et al. ${ }^{[21]}$ found that the adherence rate to HCC surveillance was only $52 \%$.

The regular follow-up group had a higher survival (43.8\% vs. 30.9\%) and smaller mean tumor size (2.72 vs. $4.59 \mathrm{~cm}$ ) than the out-of-schedule group (Table 2; $P<0.001$ ). These results are in agreement with a randomized control study that included 18,816 participants $^{[22]}$. Zhang et al ${ }^{[22]}$ found that regular surveillance leads to early detection of HCC, resulting in better survival than in those without surveillance. A recent extensive review confirms surveillance improved survival ${ }^{[23]}$. There is therefore no doubt that surveillance should be carried out in high-risk HBsAg carriers. The current Asian Pacific Association for Study of the Liver (APASL) guideline specifies that male HBsAg carriers aged greater than 40 years and females aged greater than 50 years are high-risk groups ${ }^{[7]}$. In our previous analysis, which used a subset of patients from this cohort, we found that the incidence of HCC was relatively low if the initial ALT level was lower than $2 \times$ ULN or if the patients maintained persistent normal $\mathrm{ALT}^{[23,24]}{ }^{2}$. In this study, $72.6 \%$ of chronic HBsAg carriers without HCC had initial ALT levels lower than $2 \times$ ULN, and 69.3\% had maximal ALT levels lower than $5 \times$ ULN. In contrast, $63.5 \%$ HCC patients had initial ALT levels greater than or equal to $2 \times \mathrm{ULN}$ and $71.5 \%$ had maximal ALT levels greater than or equal to $5 \times$ ULN. Therefore, we might not encourage patients with persistent ALT levels lower than $2 \times$ ULN, no cirrhosis, female gender, or age under 40 years to receive early full surveillance. Repeated negative findings during regular follow-up visits may cause the patient to feel it is less necessary to continue surveillance. For such patients, we may continue with simple ALT and AFP surveys. Full surveillance, including HBV viral load, US, elastography, or new markers may then be started once a risk factor is identified. Active call-back mechanisms focusing on these high-risk patients will be mandatory.

The prevalence of liver cirrhosis was relatively high (70.2\%) in patients with HCC. In addition, lesions resembling liver regeneration nodules were found in 47 (36.2\%) patients preceding HCC diagnosis. Of these, $16(12.3 \%)$ had three or more nodules. These preexisting findings decreased the likelihood of early diagnosis of HCC. Indeed, cirrhotic nodules have been reported as a problem in the diagnosis of small HCC $^{[25]}$. New parameters to allow the discrimination of HCC from regeneration nodules will therefore be needed ${ }^{[26-28]}$. 
More patients with HCC received treatment than those without HCC (Table 1; $P<0.001$ ). This could be because active viral replication is typically associated with $\mathrm{HCC}^{[29,30]}$. Since the treatment regimens were complicated, whether anti-HBV therapy decreased the incidence of HCC could not be evaluated in this study.

We conclude that surveillance for early diagnosis of HCC improved the survival of high-risk HBsAg carriers. Most HBsAg carriers are low-risk and can be screened using simple parameters, such as periodic AFP and liver biochemistry tests at 6-12-month intervals. When risk factors appear during this follow-up schedule, we may then add US, elastography, or other new markers for early diagnosis of HCC in high-risk patients.

\section{DECLARATIONS}

\section{Acknowledgments}

We would like to thank Uni-edit (www.uni-edit.net) for editing and proofreading this manuscript.

\section{Authors' contributions}

Designed the study and wrote the manuscript: Tai DI

Update treatment data and wrote the manuscript: Chen CJ, Tai DI

Collected and organized data: Tai J

\section{Availability of data and materials}

The data source is from Carrier Clinics of Chang Gung Memorial Hospital. Please contact the author through E-mail for information if necessary.

\section{Financial support and sponsorship}

This research was supported by the grant from Chang Gung Memorial Hospital (CMRPG3E1121 and CIRPG3H0021).

\section{Conflicts of interest}

The author declared that there are no conflicts of interest.

\section{Ethical approval and consent to participate}

Not applicable.

\section{Consent for publication}

Not applicable.

\section{Copyright}

(c) The Author(s) 2019.

\section{REFERENCES}

1 Schweitzer A, Horn J, Mikolajczyk RT, Krause G, Ott JJ. Estimations of worldwide prevalence of chronic hepatitis B virus infection: a systematic review of data published between 1965 and 2013. Lancet 2015;386:1546-55.

2 Hou J, Liu Z, Gu F. Epidemiology and prevention of hepatitis B virus infection. Int J Med Sci 2005;2:50-7.

3 Tai DI, Jeng WJ, Lin CY. A global perspective on HBV-related SNPs and evolution during human migration. Hepatol Commun 2017;1:1005-13.

4 Liaw YF, Chu CM. Hepatitis B virus infection. Lancet 2009;373:582-92.

5 Liaw YF, Tai DI, Chu CM, Lin DY, Sheen IS, et al. Early detection of hepatocellular carcinoma in patients with chronic type B hepatitis: a prospective study. Gastroenterology 1986;90:263-7. 
6 Liaw YF, Tai DI, Chen TJ, Chu CM, Huang MJ. Alpha-fetoprotein changes in the course of chronic hepatitis: relation to bridging hepatic necrosis and hepatocellular carcinoma. Liver 1986;6:133-7.

7 Omata M, Cheng AL, Kokudo N, Kudo M, Lee JM, et al. Asia-Pacific clinical practice guidelines on the management of hepatocellular carcinoma: a 2017 update. Hepatol Int 2017;11:317-70.

8 Raza A, Sood GK. Hepatocellular carcinoma review: current treatment, and evidence-based medicine. World J Gastroenterol 2014;20:4115-27.

9 Tsay PK, Tai DI, Chen YM, Yu CP, Wan SY, et al. Impact of gender, viral transmission and aging in the prevalence of hepatitis B surface antigen. Chang Gung Med J 2009;32:155-64

10 Cheng HT, Chang YH, Chen YY, Lee TH, Tai DI, et al. AFP-L3 in chronic liver diseases with persistent elevation of alpha-fetoprotein. J Chin Med Assoc 2007;70:310-17.

11 Choi J, Kim GA, Han S, Lee W, Chun S, et al. Longitudinal assessment of three serum biomarkers to detect very early stage hepatocellular carcinoma. Hepatology 2019;69:1983-94.

12 Xie H, Xue YQ, Liu P, Zhang PJ, Tian ST, et al. Multi-parameter gene expression profiling of peripheral blood for early detection of hepatocellular carcinoma. World J Gastroenterol 2018;24:371-8.

13 Ng CKY, Di Costanzo GG, Terracciano LM, Piscuoglio S. Circulating cell-free DNA in hepatocellular carcinoma: current insights and outlook. Front Med (Lausanne) 2018;5:78.

14 Pezzuto F, Buonaguro L, Buonaguro FM, Tornesello ML. The role of circulating free DNA and MicroRNA in non-invasive diagnosis of HBV- and HCV-related hepatocellular carcinoma. Int J Mol Sci 2018;19:E1007.

15 Zacharakis G, Aleid A, Aldossari KK. New and old biomarkers of hepatocellular carcinoma. Hepatoma Res 2018;4:65.

16 Bruix J, Sherman M, Llovet JM, Beaugrand M, Lencioni R, et al. Clinical management of hepatocellular carcinoma. Conclusions of the Barcelona-2000 EASL conference. European Association for the Study of the liver. J Hepatol 2001;35:421-30.

17 Fattovich G, Bortolotti F, Donato F. Natural history of chronic hepatitis B: special emphasis on disease progression and prognostic factors. J Hepatol 2008;48:335-52.

18 17Lin CL, Kao JH. Risk stratification for hepatitis B virus related hepatocellular carcinoma. J Gastroenterol Hepatol 2013;28:10-7.

19 Bruix J, Sherman M. Management of hepatocellular carcinoma: an update. Hepatology 2011;53:1020-2.

20 Ronot M, Pommier R, Dioguardi Burgio M, Purcell Y, et al. Hepatocellular carcinoma surveillance with ultrasound-costeffectiveness, high-risk populations, uptake. Br J Radiol 2018;91:20170436.

21 Wang C, Chen V, Vu V, Le A, Nguyen L, et al. Poor adherence and low persistency rates for hepatocellular carcinoma surveillance in patients with chronic hepatitis B. Medicine (Baltimore) 2016;95:e4744.

22 Zhang BH, Yang BH, Tang ZY. Randomized controlled trial of screening for hepatocellular carcinoma. J Cancer Res Clin Oncol 2004;130:417-22.

23 Chen K, Chang PE, Goh GBB, Tan CK. Surveillance for hepatocellular carcinoma - current status and advances. Hepatoma Res 2018;4:72.

24 Tai DI, Lin SM, Sheen IS, Chu CM, Lin DY, et al. Long-term outcome of HBeAg-negative HBsAg carriers in relation to changes of alanine aminotransferase levels over time. Hepatology 2009;49:1859-67.

25 Tai DI, Tsay PK, Chen WT, Chu CM, Liaw YF. Relative roles of HBsAg seroclearance and mortality in the decline of HBsAg prevalence with increasing age. Am J Gastroenterol 2010;105:1102-9.

26 Rode A, Bancel B, Douek P, Chevallier M, Vilgrain V, et al. Small nodule detection in cirrhotic livers: evaluation with US, spiral CT, and MRI and correlation with pathologic examination of explanted liver. J Comput Assist Tomogr 2001;25:327-36.

27 Jo PC, Jang HJ, Burns PN, Burak KW, Kim TK, et al. Integration of contrast-enhanced US into a multimodality approach to imaging of nodules in a cirrhotic liver: how I do it. Radiology 2017;282:317-31.

28 Inchingolo R, De Gaetano AM, Curione D, Ciresa M, Miele L, et al. Role of diffusion-weighted imaging, apparent diffusion coefficient and correlation with hepatobiliary phase findings in the differentiation of hepatocellular carcinoma from dysplastic nodules in cirrhotic liver. Eur Radiol 2015;25:1087-96.

29 Yin CQ, Yuan CH, Qu Z, Guan Q, Chen H, et al. Liquid biopsy of hepatocellular carcinoma: circulating tumor-derived biomarkers. Dis Markers 2016;2016:1427849.

30 Tai DI, Changchien CS, Hung CS, Chen CJ. Replication of hepatitis B virus in first-degree relatives of patients with hepatocellular carcinoma. Am J Trop Med Hyg 1999;61:716-9. 